\title{
Recent advances on Toll-like receptor 4 modulation: new therapeutic perspectives
}

\author{
Lenny Zaffaroni ${ }^{1} \&$ Francesco Peri*,1 \\ ${ }^{1}$ Department of Biotechnology \& Biosciences, University of Milano-Bicocca; Piazza della Scienza, 2; 20126 Milano, Italy \\ *Author for correspondence: Tel.: +39 02 64483453; francesco.peri@unimib.it
}

\begin{abstract}
Activation or inhibition of TLR4 by small molecules will provide in the next few years a new generation of therapeutics. TLR4 stimulation (agonism) by high-affinity ligands mimicking lipid A gave vaccine adjuvants with improved specificity and efficacy that have been licensed and entered into the market. TLR4 inhibition (antagonism) prevents cytokine production at a very early stage; this is in principle a more efficient method to block inflammatory diseases compared to cytokines neutralization by antibodies. Advances in TLR4 modulation by drug-like small molecules achieved in the last years are reviewed. Recently discovered TLR4 agonists and antagonists of natural and synthetic origin are presented, and their mechanism of action and structure-activity relationship are discussed.
\end{abstract}

First draft submitted: 4 August 2017; Accepted for publication: 9 November 2017; Published online: 30 January 2018

Keywords: CD14 • MD-2 $\bullet$ natural compounds $\bullet$ structure-activity relationship $\bullet$ synthetic compounds $\bullet$ TLR4

\section{TLR4-associated pathologies \& possible TLR4-based therapies}

Toll-like receptors (TLRs) belong to the class of pattern recognition receptors and are the first line of defense from invading pathogens in humans and higher animals. TLRs recognize pathogen-associated molecular patterns (PAMPs), thus activating inflammation and innate immunity response [1]. Among TLRs, TLR4 is the specific sensor of lipopolysaccharide (LPS), one of the molecular components of Gram-negative outer membrane, and its truncated versions lipooligosaccharide (LOS) and lipid A, collectively termed as endotoxin [2,3]. TLR4 also recognizes endogenous molecules, called damage-associated molecular pattern (DAMPs) molecules, released by injured tissues and necrotic cells [4]. TLR4-mediated inflammation, triggered by PAMPs and DAMPs, is involved in several acute and chronic diseases. Hence, TLR4 is a key receptor on which both noninfectious and infectious stimuli converge to trigger a proinflammatory response. TLR4 can then have an inflammatory or repair role. Inflammation, in general, has a protective function, and TLR 4 can play an important role in this context; in particular by activating the induction of specific resolution pathways that restore tissue integrity and function [5]. However, when the TLR4 inflammatory response is not regulated, it can be harmful for the organism. In several diseases with microbial (Gram-negative infections) or nonmicrobial etiology (ischemia/reperfusion injury, sterile and chronic inflammations, autoimmune diseases and neuroinflammation), TLR4 activation and signaling contribute to disease progression [6].

The most severe disease deriving from TLR4 excessive activation by PAMPs is sepsis. Sepsis is a dysregulated response of the host organism to outer pathogens, which leads to acute life-threatening organ dysfunction [7]. The global incidence of this syndrome accounts for 437 per 100,000 person-years between the years 1995 and 2015, according to retrospective analysis of an international database [8]. In western countries, mortality in patients with severe sepsis is $20-50 \%$, and if there is no organ dysfunction it can be diminished (less than 20\%). Septic shock with increased LPS levels in blood, overexpression of proinflammatory cytokines, activation of blood coagulation system and accumulation of fibrinogen degradation products leads to a violation of local and general hemodynamics and endothelial dysfunction via TLR4 signaling pathway.

Sepsis is one of the possible complications of severe influenza. The most typical bacterial species complicating disease are Streptococcus pneumoniae, Pseudomonas aeruginosa, Acinetobacter species, Staphylococcus aureus as well as Enterobacteriaceae species, Aspergillus species and others [9-11]. Sepsis is the most important example of pathology caused by TLR 4 excessive and dysregulated activation by LPS that could be efficiently blocked by using TLR 4 
antagonists such as Eritoran [12]. However, despite its efficacy in contrasting acute sepsis in animal models, Eritoran failed to pass clinical Phase III trials on septic patients [13].

Other pathologies are associated to DAMP/TLR4 signaling, for instance TLR4 plays a key role in the CNS neurodegeneration [14]. In CNS, TLRs activation can be either detrimental (neuroinflammation and neurodegeneration) or beneficial (tissue repair), or have a mixed, still not fully understood effect [15].

In the context of DAMP-associated pathologies, the inhibition of TLR4 stimulation by endogenous factors could be used to contrast a wide range of inflammatory disorders. These pathologies are caused by the release of reactive nitrogen or oxygen species and inflammatory cytokines following 'sterile inflammations' induced by DAMPs. In this context, TLR4 is a molecular target related to a broad spectrum of modern day diseases including asthma [16], allergies [17], chronic inflammations, autoimmune disorders, CNS diseases linked to neuroinflammation and cancer [18].

TLR4 has also been suggested as a promising therapeutic target for drug abuse [19] and major depressive disorder [20], as well as amyotrophic lateral sclerosis [21]. Possible application of synthetic TLR4 antagonists in the treatment of neuropathic pain has also been shown [22,23]. From a molecular point of view, TLR4 activation by LPS is based on the successive interaction of LPS with LBP [24] that transfers a single molecule of LPS from aggregates in solution to CD14 [25], that in turns shuttles LPS to the TLR4/MD-2 [26] complex. The whole molecular process ends with the formation of the (LPS/MD-2/TLR4) 2 complex on plasma membrane [27]. The complex transmits the signal in the cell by recruiting the MyD88 and the adapter MAL (MyD88-dependent pathways and production of a number of proinflammatory cytokines) or TRIF (MyD88-independent pathways and production of interferons) [1-3]. On the other hand, LOS can directly interact with the TLR4/MD-2 complex and activate TLR4 signaling without the participation of LBP and CD14 co-receptors [28,29].

Molecules active as TLR 4 antagonists, Eritoran or TAK-242, have failed to block acute sepsis $[13,30]$. However, a new perspective is holding promise in using TLR 4 antagonists to block TLR 4 activation by DAMPs in chronic inflammatory diseases and sterile inflammations. DAMPs are released from inflamed and damaged tissues [31]. Unequivocal evidence that DAMPs are specific TLR4 agonists remains to be provided in some cases, because in biochemical experiments aimed at identifying endogenous TLR4 agonists, the activation of TLR 4 pathway could actually derive from endotoxin contamination in DAMPs. While the molecular mechanisms of endotoxin (LPS and LOS) TLR4 activation are well understood [18], the mechanisms of DAMP/TLR 4 signaling are only partially known. The high chemical diversity of recently discovered endogenous TLR4 ligands seems to be incompatible with the molecular specificity of TLR4 receptor. A recent critical paper on this subject recommended that some requirements are respected when assessing TLR 4 agonism [32]. The most important condition is that the putative agonist should engage both TLR4 and MD-2 receptors for signaling and directly interacts with MD-2 and/or CD14 receptors [32].

As a follow-up of our previous reviews on this topic [18], we present here last achievements on the discovery of natural and synthetic molecules that modulate TLR4 activity as agonists or antagonists. We focus on small molecules (MW less than $1 \mathrm{KDa}$ ) that have been validated as TLR4 ligands according to the rules above mentioned, and on their mechanism of action.

In the first part, new synthetic TLR4 modulators are reported, in the second part are described compounds of natural origin.

\section{Synthetic TLR4 modulators}

\section{From lipid A mimetics to other compounds}

The rational design of lipid A variants and analogs afforded several TLR 4 agonists and antagonists with glycolipid structures that have been reviewed by us [18] and others [33,34]. All these structures include the chemical determinant (pharmacophore) essential for MD-2 recognition and binding: a disaccharide core (GlcNAc-GlcNAc) with six lipid chains, and two negatively charged phosphates or phosphates bioisostere groups, at positions C1 and C4' of the disaccharide. These are Eritoran [35], monophosphoryl lipid A (MPLA) [36] and aminoglycosides [37] lipid A mimetics. Other lipid A mimetics are based on a monosaccharide scaffold derived from lipid X, a biosynthetic precursor of lipid A, with activity as TLR 4 antagonist [38]. All these compounds are sparingly soluble and have poor pharmacokinetic in vivo. The new tendency is therefore to explore new chemical structures, often derived from natural compounds, to obtain new TLR4 ligands with improved solubility and pharmacokinetics. We report here on TLR4 ligands and modulators recently discovered. Generally, the chemical structures of these molecules are 
different from that of lipid A. Very often these compounds are structurally derived from natural compounds that have been found active on TLR 4 or are the results of a random screening of libraries of compounds.

\section{Glycolipid-based compounds}

\section{Fatty acid esters of monogalactosyldiacylglycerol}

Fatty acid (FA) esters of monogalactosyldiacylglycerol (MGDG) are essential components of the thylakoid membrane in chloroplasts and dilinolenoyl MGDG has been reported to show anti-inflammatory effects in human peripheral blood neutrophils [39].

In order to understand the mechanism of action of these molecules, a derivative of dilinolenoyl MGDG, a bifunctional, MGDG-based probe (molecule 1; Figure 1) has been synthesized.

$\mathbf{1}$ contains two chemical functions, one reactive toward nucleophile groups in the target protein, another, the alkyne function, forms triazole adducts by reacting with azide groups (click reaction). 1 inhibited LPS-mediated TLR4 activation in a concentration-dependent manner in cells. Consistently with other studies [40], $\mathbf{1}$ showed strong inhibition of $\mathrm{p} 38$ phosphorylation without influencing the total cell number being a more potent inhibitor than parent MGDG.

Endogenous proteome labeling followed by pull down/LC-MS/MS showed that compound $\mathbf{1}$ mainly interacts with the TLR4/MD-2 complex. By molecular modeling it has been calculated the lowest-energy binding pose of $\mathbf{1}$ with the TLR4/MD-2 complex. $\mathbf{1}$ binds to MD-2 similarly to Eritoran, both FA chains of $\mathbf{1}$ insert into the hydrophobic pocket of MD-2, while the sugar part interacts with residues of the MD-2 cavity rim. The binding mode between 1 and TLR4/MD-2 shows that FA chains forms favorable hydrophobic interactions with human TLR4 (hTLR4) residues including F440, F463, L444, K388 and Q436, which facilitate the subsequent formation of a covalent bond with TLR4.

Mutational studies showed that two residues of hTLR4, namely F440 and F463, are essential for binding to 1 . Previous functional studies have identified that both F440 and F463 are required for cell activation by LPS. These mutational data reinforce the vision of a mechanism of action of MGDG based on direct competition with LPS for MD-2 binding.

\section{Trimannoside glycolipid conjugates}

Trimannoside glycolipid conjugates (MGCs; 2; Figure 1) are formed by a branched core linked to three mannose units through triethylene glycol linkers and to a saturated or unsaturated $\mathrm{C}_{24}$ lipophilic chain. MGCs selectively blocked TLR4-mediated activation of human monocytes and monocyte-derived dendritic cells (DCs) by LPS [41].

The mechanism of action of MGCs is unique: while these compounds inhibit both MyD-88 and TRIF-mediated intracellular pathways deriving from TLR4 activation and also block NF- $\kappa \mathrm{B}$ activation and nuclear translocation, they do not directly compete with LPS for MD-2/TLR4 binding. The action of MGCs is therefore not based on a direct competition with LPS for CD14 and/or MD-2 binding as in the case of the majority of other TLR4 antagonists.

2 has a disruptive action on lipid raft-located GPI-anchored proteins, CD1a and CD14, and induces their rapid cell internalization. Thus, the plasma membrane may be a principal target through which the modulatory effects of MGCs are mediated. In the presence of LPS, 2 could prevent CD14 and TLR4 co-localization. These results suggest that the inhibitory action of $\mathbf{2}$ and other MGCs is mediated by their effect on the cell membrane: they alter lipid raft composition, interfere with the clustering of CD14 and TLR4 in rafts, leading to impaired receptor complex formation and blocking of signal transduction. Moreover, selective deprivation of CD14 from the cellular membrane, and subsequent impairment of TLR4/MD-2 complex formation could be a new mechanism for TLR 4 antagonism. A similar mechanism of action has been recently described for monosaccharide 3, a synthetic TLR4 antagonist [42].

\section{Monosaccharide derivatives}

The diphosphorylated diacylated monosaccharide FP7 (compound 3; Figure 1), is a synthetic molecule with potent TLR4 antagonist action both in cells and in animals [42,43]. Monosaccharide 3 inhibits the LPS-stimulated, TLR4-dependent secretion of proinflammatory cytokines (IL-6, IL-8 and MIP-1 $\beta$ ) by monocytes and DCs and prevents DCs maturation upon TLR4 activation by LPS [43]. The mechanism of action of $\mathbf{3}$ is based on the direct competition with LPS for MD-2 binding probably reinforced by direct binding to CD14 co-receptor. ${ }^{1} \mathrm{H}$ NMR experiments demonstrated that after addition of MD-2, the signals of the two FA chains $\left(\mathrm{C}_{14}\right)$ of $\mathbf{3}$ is attenuated [42] 


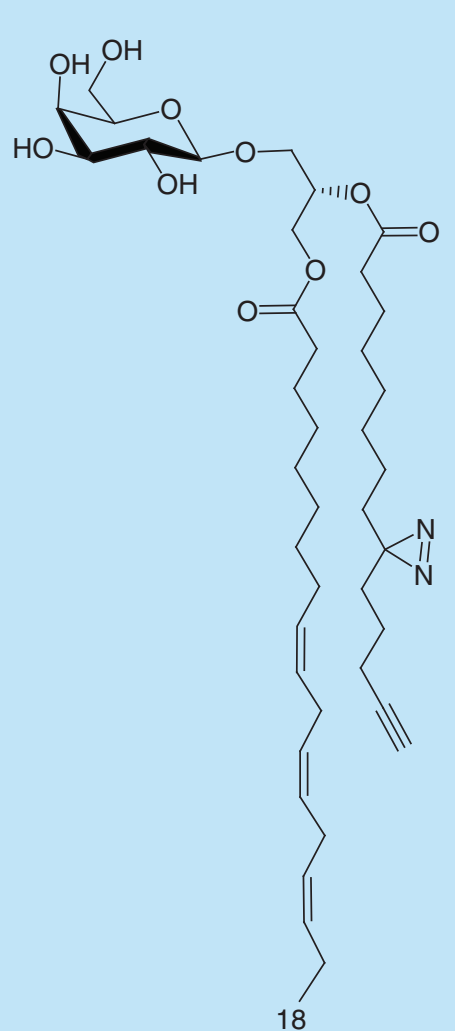

1

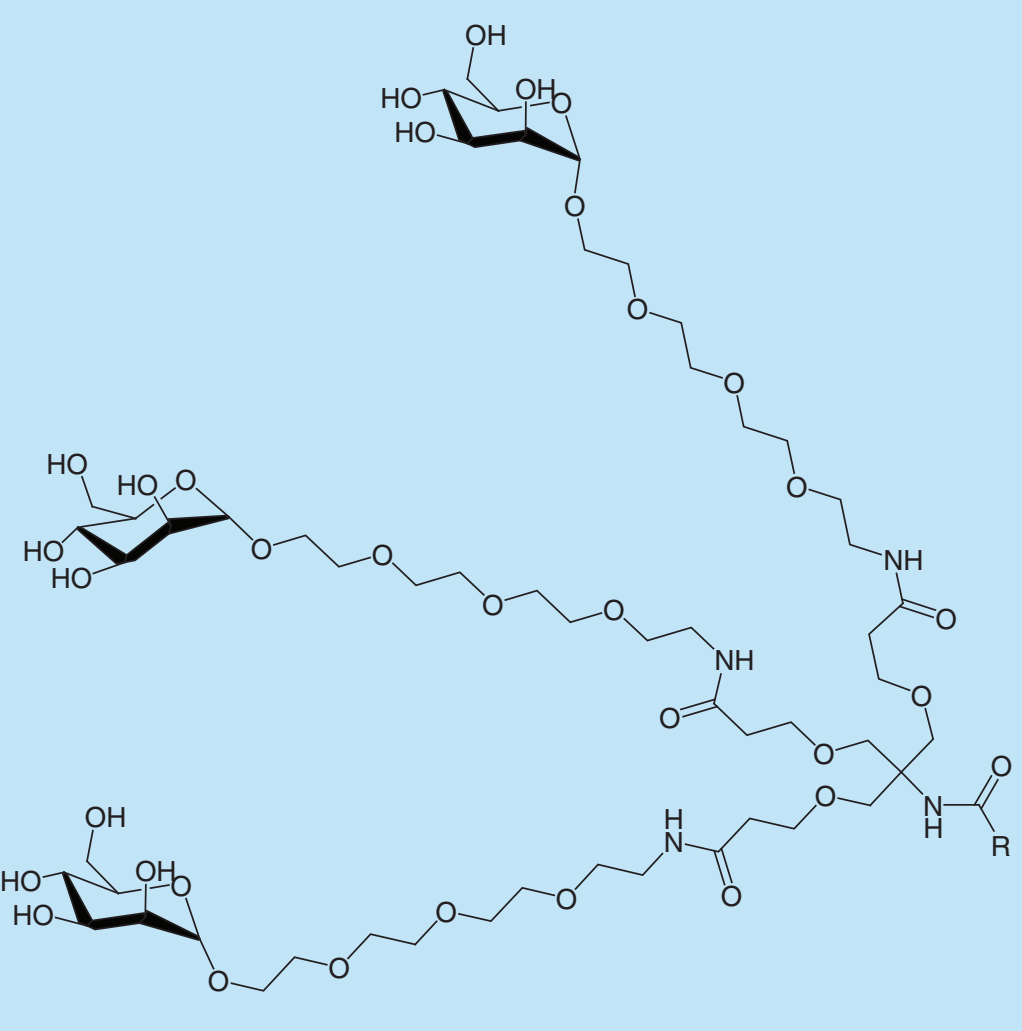

2
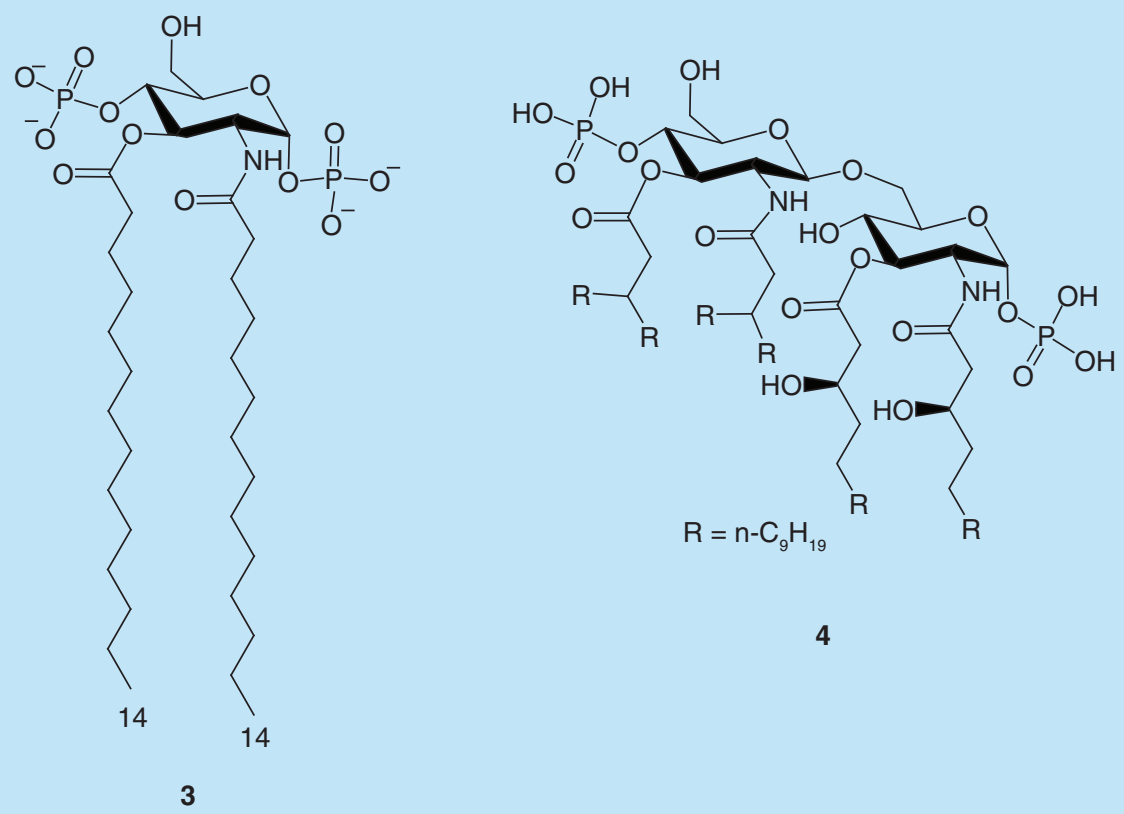

4

Figure 1. Recently discovered synthetic, glycolipid-based TLR4 modulators. (1) bifunctional MGDG probe, (2)

trimannoside glycolipid conjugates, $\mathrm{R}$ = saturated or unsaturated $\mathrm{C}_{24}$ lipophilic chain, (3) monosaccharide FP7 and (4) vizantin-lipid A conjugate.

MGDG: Monogalactosyldiacylglycerol. 
thus suggesting that lipophilic chains are the main interacting units of compound 3 with MD-2 hydrophobic pocket.

The capacity of 3 to induce CD14 and TLR4/MD-2 complex internalization in murine cells was analyzed [42]. Cell exposure to compound 3 caused endocytosis of CD14 but not of the TLR4/MD-2 complex. These results suggest that 3 interact directly with CD14, causing its internalization and deprivation of CD14 on plasma membrane could explain the antagonist action of $\mathbf{3}$.

In vitro binding data with purified, functional MD-2 (Facchini FA et al., Unpublished Data) show that 3 is able to displace LPS from human MD-2 (hMD-2), and binds to hMD-2 with an affinity $\left(\mathrm{K}_{\mathrm{D}}\right)$ in the low micromolar range.

The TLR 4 antagonist Eritoran has been recently used to contrast influence lethality in animal models [44]. The infection of some influenza strains induces a TLR4-dependent cytokine storm similar to septic shock. Abnormal TLR4 stimulation is due to the release of endogenous TLR4 agonists (DAMPs) deriving form damage of lung tissue, such as oxidized phospholipids from infected tissue or circulating HMGB1 protein. The DAMP/TLR4 signaling related to influenza inflection is inhibited by Eritoran [44].

Similar to Eritoran, 3 selectively blocked DAMP/TLR4 signaling in animal models, protected mice from PR8 influenza virus-induced lethality and reduced proinflammatory cytokine gene expression in the lungs and acute lung injury (ALI) [43]. In a proof-of-concept experiment, compound 3 inhibited in a dose-dependent manner the DCs activation by endogenous HMGB1 [43]. This suggests that the mechanism of action of $\mathbf{3}$ in blocking influenza lethality could be based on inhibition of HMGB1/TLR4 signaling. The good bioavailability of $\mathbf{3}$, together with its high water solubility, lack of toxicity and selective TLR4 antagonist action, make it a new drug hit targeting TLR4-related microbial and sterile inflammatory diseases.

\section{Lipid A mimetic with Vizantin-like branched chains}

In a recent study, it was shown that the trehalose derivative named Vizantin (6,6'-bis-O-(3-nonyldodecanoyl)$\alpha, \alpha^{\prime}$-trehalose) displays adjuvant activity based on TLR4 agonism and binding to the TLR4/MD-2 complex. Based on the known biological activity of lipid A and Vizantin, the authors projected a novel bifunctional glycolipid, that combines the structural features of Vizantin (namely, the two branched FA chains 3-nonyldodecanoic acid, 3NDDA) with the glucosamine core of lipid A (compound 4; Figure 1) [45]. Disaccharide 4 potently antagonizes the LPS/TLR4 signaling pathway in a concentration-dependent manner, in human THP-1 cells, with an IC 50 of $3.8 \mathrm{nM}$. Molecular docking simulations with MD-2 and MD-2/TLR4 suggest that the two branched NDDA chains of compound $\mathbf{4}$ insert into MD-2 binding cavity thus stabilizing the complex by hydrophobic interaction with the receptor.

\section{Nonglycolipid TLR4 modulators Neoseptins}

The screening of 90,000 compounds tested for their ability to activate TNF- $\alpha$ in mouse peritoneal macrophages, led to the identification of a new TLR 4 agonist, Neoseptin-1. Structure-activity relationship studies allowed to improve the design of this type of LPS-binding peptide mimetics, leading to Neoseptin-3 hit compound (5; Figure 2) [46].

5 is a peptidomimetic with no structural similarity to LPS that activates mouse TLR4 (mTLR4). In vitro dose response experiments demonstrated that molecule 5 activates TLR 4 in different mouse cells. Induction of TNF- $\alpha$ by 5 was completely abrogated in TLR4- or MD-2-deficient mouse macrophages, while CD14-deficient macrophages that do not respond to LPS, still produced TNF- $\alpha$ in response to 5.

5 activates mouse TLR4/MD-2 independently of CD14 and triggers canonical MyD88 and Toll IL-1 receptor domain-containing adaptor inducing IFN- $\beta$ (TRIF)-dependent signaling. On the other hand, $\mathbf{5}$ is unable to stimulate human TLR4, as turned out from experiments on human THP-1 monocytes. This supported the idea that the TLR4 agonist activity of $\mathbf{5}$ is species-specific, with a preference toward mouse MD-2 (mMD-2). The direct interaction of 5 with mTLR4/MD-2 heterodimer with formation of a complex was observed by NMR binding experiments and, more directly, by $\mathrm{x}$-ray analysis of the complex. The crystal structures of TLR4/MD-2 complex in the 'apo' form (i.e., without any ligand), in complex with lipid A, and with $\mathbf{5}$ were compared. In all the three different cases studied, the structure of the mTLR4/MD-2 complexes presented similar conformations. While having completely different chemical structures, lipid A and $\mathbf{5}$ are capable of inducing similar conformational changes of the MD-2 Phe126 loop region, the typical conformational switch induced by agonists. Interestingly, two molecules of $\mathbf{5}$ were found asymmetrically bound to MD-2 cavity in the complex with mTLR4/MD-2. The two 


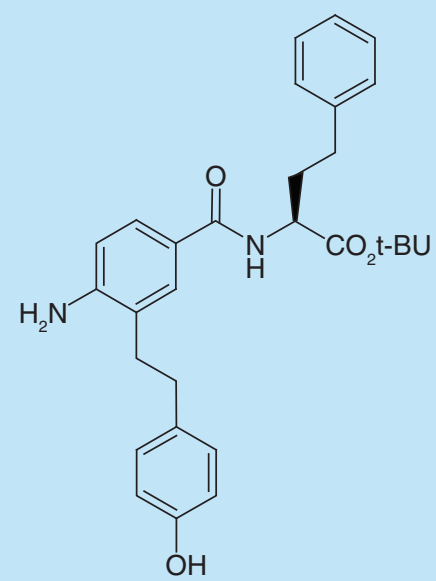

5<smiles>O=S(=O)(O)c1cc(N=C=S)ccc1/C=C/c1ccc(N=C=S)cc1S(=O)(=O)O</smiles>

7<smiles>O=C(O)Cc1ccc(C(C(=O)NC2CCCC2)N(C(=O)c2cc(-c3ccccc3)n(-c3ccccc3)n2)c2ccc3c(c2)CCC3)cc1</smiles>

6<smiles>COc1ccc(C(=O)/C=C/c2cc(OC)c(OC)c(OC)c2)cc1OC</smiles>

Figure 2. Nonglycolipid synthetic TLR4 modulators. (5) Neoseptin-3, (6) compound AZ617, (7) chalcone compound 20, and (8) 4,4'-Diisothiocyanostilbene-2,2'-disulfonic acid.

molecules occupy different sites at the rim of MD-2 binding cavity and interact with the second TLR4 molecule (TLR4 $\left.{ }^{*}\right)$ thus promoting TLR4 dimerization and activation. The final activated complex (TLR4/MD-2/5 2$)_{2}$ contains four molecules of $\mathbf{5}$. This study provides new and interesting information on how a molecule different from lipid A can promote the formation of the activated TLR4/MD-2 heterodimer by binding MD-2 and TLR4*. The dissociation between CD14 function and MyD88/TRIF signaling pathways is reminiscent of the mechanism of action of other small-molecular TLR4 agonists, including Ugi compounds presented in the next paragraph. However, the lack of activity on human TLR4 receptor system limits the clinical development of Neoseptins as TLR4-directed therapeutics.

\section{Ugi compounds}

A panel of small molecules with TLR 4 agonist activity was synthesized through a 4-component Ugi condensation reaction (the more potent molecule being AZ617, compound $\mathbf{6}$ in Figure 2) [47]. The majority of the synthetic Ugi compounds presented TLR4 agonist in HEK-293 cells transfected with hTLR4/hMD-2/hCD14 while were almost inactive in stimulating HEK cells transfected with mTLR4/mMD-2/mCD14. Species-specific TLR4 activation is due to higher affinity binding of Ugi compounds to hMD-2 than to mMD-2. By comparing the activity of Ugi compounds in HEK 'hybrid' transfectants with a combination of human and mouse receptors, namely hTLR4/mMD-2 and mTLR4/hMD-2, authors observed that Ugi compounds turned out to be more active in the transfectants containing hMD-2. The capacity of Ugi compounds to stimulate human TLR4 receptor system was also confirmed on human peripheral blood mononuclear cells (PBMCs). The preference of human TLR4 receptor system over murine is however peculiar, because LPS and MPLA, show an opposite preference for murine receptors giving a stronger activation in cells expressing mMD-2. Another important difference with LPS and lipid A-derived agonists is that the TLR4 activation mediated by compound $\mathbf{6}$ is CD14-independent. In silico studies 
showed two possible sets of binding poses (with almost identical energy) of Ugi compounds into the hydrophobic binding pocket of MD-2.

As Ugi compounds have a TLR4 agonist preference toward human species, and a better water solubility compared with other lipid A analogs, making them a good starting point for the development of adjuvants that can activate TLR4 in cells with physiological low levels of CD14, such as DCs.

\section{Phospholipids}

Lung alveolar epithelium secretes a mixture of several proteins and lipids called pulmonary surfactants, which have important immunoregulatory properties, able of regulating the innate immune system by competitively antagonizing ligand-dependent activation of TLR 4 [48]. Phospholipids account for $90 \%$ of pulmonary surfactants, and it has been reported that minor components of pulmonary surfactants, palmitoyl-oleoyl-phosphatidylglycerol (POPG) and phosphatidylinositol, are capable of inhibiting LPS-induced inflammatory responses in U937 cells, primary rat alveolar macrophages, and primary human alveolar macrophages by blocking LPS-induced phosphorylation of

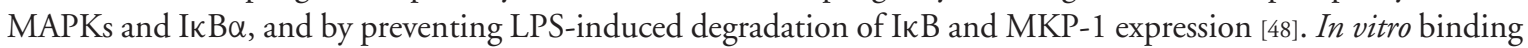
studies showed that POPG and phosphatidylinositol bind to LBP, CD14, and MD-2 in a concentration-dependent manner. A recent study screened a library of POPG variants, with the aim to understand the phospholipids' structural features that dictate the antagonistic activity against TLR2 and TLR4 [49]. In summary, POPG analogs with polar head group modifications can antagonize TLR2 and TLR4 activation. POPG analogs strongly bind to CD14 and MD-2 proteins, thus causing TLR4 inhibition. The structural plasticity necessary for TLRs modulation open up possibilities for the design of new POPG-like molecules that can possess individual TLRs specificity.

\section{Chalcone derivatives}

Several chalcone derivatives that contain the moiety of (E)-4-phenylbut-3-en-2-one, considered the core structure of currently known MD-2 inhibitors of natural origin such as curcumin, caffeic acid phenethyl ester and 1dehydro-10-gingerdione, have been designed and synthesized in a recent study [50]. Among all the synthesized chalcone compounds, compound 7 (Figure 2) turned out to be the more potent in antagonizing the TLR4 pathway both in vitro and in vivo. Fluorescence spectroscopy experiments showed that 7 competitively inhibits the interactions between MD-2 and LPS, and also between TLR4 and MD-2. The direct interaction between 7 and MD-2 was confirmed by surface plasmon resonance experiments. Computational studies shown that in the lower energy binding pose, 7 would interact with MD-2 residues Arg90 and Tyr102, by forming two hydrogen bonds. This finding was experimentally confirmed by using MD-2 mutants [51]. 7 is also able to attenuate LPS-induced lung injuries, in particular by diminishing pulmonary inflammation and by preventing the interaction between MD-2 and TLR4 in lung tissue. Similar to other TLR4 antagonists as Eritoran and monosaccharide 3, chalcone derivative 7 can be considered a hit compound for the development of drugs for the treatment of ALI and other TLR4-dependent syndromes induced by pathogens infections.

\section{Calcineurin inhibitors}

Calcineurin inhibitors (CNIs) cyclosporine A and tacrolimus are active in increasing the production of proinflammatory cytokines and endothelial activation markers through TLR4 activation in cultured murine endothelial and vascular smooth muscle cells as well as in ex vivo cultures of murine aortas [52]. Data showed that CNIs were unable to induce inflammation in aortas from Tlr $4^{-1-}$ mice and after pharmacological inhibition of TLR4 in endothelial cells. However, further research is required to clarify the exact mechanisms of action by which CNIs are able to activate the TLR4 pathway.

\section{4,4'-Diisothiocyanostilbene-2,2'-disulfonic acid}

The anti-inflammatory effects of 4,4'-Diisothiocyanostilbene-2,2'-disulfonic acid (compound $\mathbf{8}$, Figure 2), a chloride channel blocker, were investigated in a recent study [53]. 8 significantly inhibits LPS-induced release of proinflammatory cytokines in vitro and in vivo studies, downregulating the inflammatory cytokines via inhibition of the TLR4/NF- $\mathrm{kB}$ pathway, with a clear indication that $\mathrm{ClC}-3$ (a volume-activated chloride channel protein) is involved in the inhibitory effect of $\mathbf{8}$. This study showed that abrogating ClC-3 inhibits the LPS-induced inflammatory response by inhibiting the TLR4/NF-KB pathway in vivo and in vitro, and this mechanism could represent a novel way for TLR4/NF-KB inhibition based on chloride channels. 


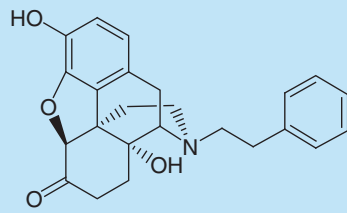

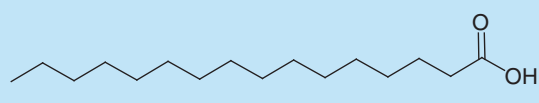

10

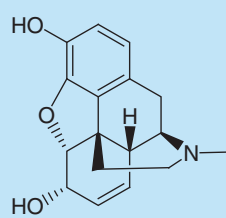

11

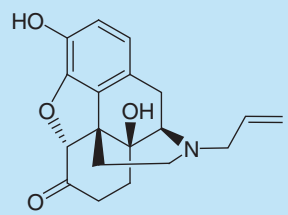

12

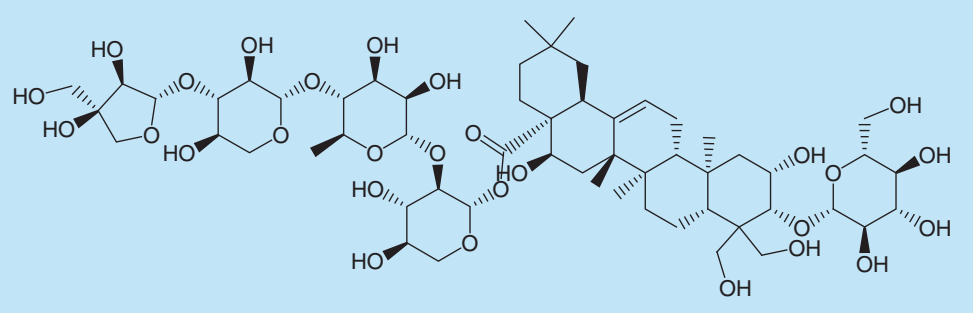

13

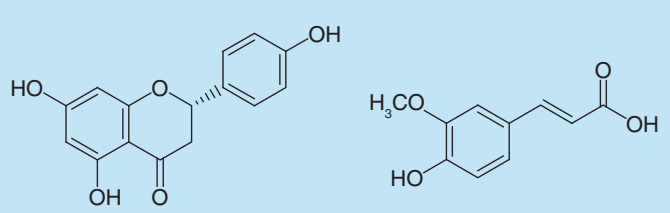

14

15

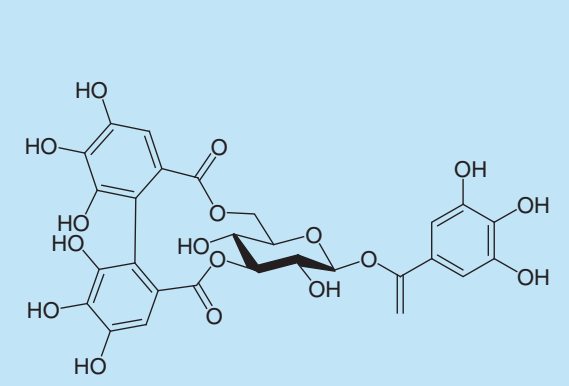

16

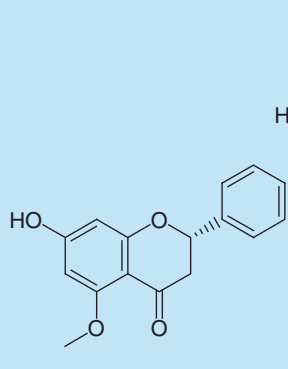

17

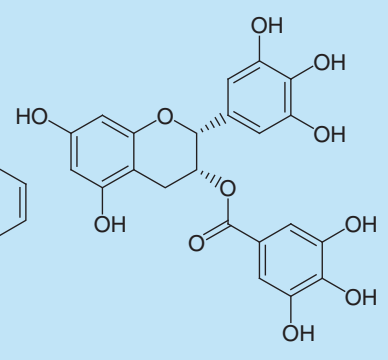

18

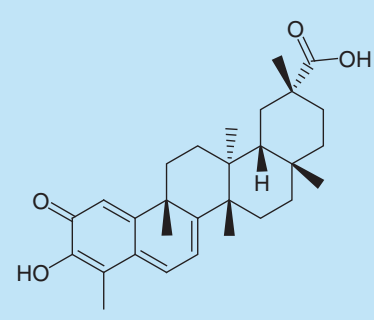

19

Figure 3. TLR4 modulators of natural origin. (9) N-phenethyl-noroxymorphone, (10) palmitic acid, (11) morphine, (12) naloxone, (13) platycodin, (14) naringenin, (15) ferulic acid, (16) corilagin, (17) alpinetin, (18) epigallocatechin-3-gallate and (19) celastrol.

\section{Morphine derivatives}

Compounds based on simplified morphine analogues $(+)$-naltrexone and $(+)$-noroxymorphone have been designed, synthesized and their TLR4 antagonist activities have been evaluated by their effects on inhibiting LPS induced TLR4 downstream nitric oxide production in microglia BV-2 cells [54]. Among all the compounds synthetized, the $N$-phenethyl-noroxymorphone (compound 9; Figure 3) was the most potent TLR4 antagonist with an $\mathrm{IC}_{50}$ of $1.4 \mu \mathrm{M}$, and no cell cytotoxicity. This analog also showed in vivo efficacy in potentiating morphine analgesia, but further research is required to investigate its exact mechanism of TLR4 inhibition.

\section{TLR4 modulators of natural origin}

Plant secondary metabolism provides a vast source of chemically different bioactive and pharmacologically active compounds. Traditional Chinese and Indian medicine use a variety of herbs that are rich in molecules that very likely act as TLR 4 modulators [55]. TLR4 activation or inhibition mediated by herbal extracts promoted a vast area of research which focuses on the molecular mechanism of action of these TLR4 modulators [56].

\section{Saturated FAs (palmitic acid)}

The molecular mechanism explaining the inflammatory action of palmitic acid (compound 10, Figure 3; the most abundant circulating saturated fatty acids [SFAs]), has recently been proposed [57]. It has been observed that $\mathbf{1 0}$ induces myocardial inflammatory injury and dysfunction through MD-2 in mouse and cell culture experimental models. The paper presented studies of purified protein-ligand interactions indicating that $\mathbf{1 0}$ directly binds to MD-2, supporting a mechanism of canonical, MD-2-dependent, TLR4 activation and signaling. However, the MD-2 binding affinity of $\mathbf{1 0}$ measured by surface plasmon resonance turned out to be very low 
(mM range). 10 is responsible for the production of proinflammatory cytokines in myocardial tissue, causing cardiac tissue remodeling and cardiac dysfunction in mice with hyperlipidemia and/or obesity. In addition, murine cardiomyocytes and macrophages acquired an MD-2-dependent proinflammatory phenotype when challenged with 10. The computational docking simulation results also supported the hypothesis of direct MD-2/10 binding and predicted that three molecules of $\mathbf{1 0}$ can accommodate into the MD-2 binding cavity. The resulting MD-2/10 complex appeared to be relatively stable, with appropriate positioning of the FAs.

Interestingly, the findings from this study supported the idea that the ability of $\mathbf{1 0}$ (or other SFAs) to activate TLR4 signaling was likely attributed to its saturated nature. The change to unsaturated FAs on the lipid A moiety of LPS results in a complete loss of TLR 4 agonist activity, and the unsaturated FA chain plays an important role in MD-2 binding of the antagonist Eritoran [58]. The authors found that, despite the binding to MD-2, unsaturated FAs did not induce TLR4/MD-2 complex formation nor activate downstream TLR4 signaling. Unsaturated FA was unable to induce cardiac inflammatory phenotype both in vitro and in vivo and did not significantly contribute to myocardial remodeling and injury in obesity. The observation that unsaturated FAs bound MD-2 suggests an intriguing possibility that unsaturated FAs may competitively inhibit SFAs to modulate TLR4 signaling response and chronic inflammation. These results underscore MD-2 as a necessary protein in SFA-mediated myocardial inflammatory injury.

\section{Morphine \& opioids}

The molecular mechanism of TLR4 stimulation by morphine and opioids has been investigated, and presents several common points with LPS activation [59]. In a recent paper the structural dynamics of the opioid-bound activation mechanism of TLR4/MD-2 complex has been studied using various computational tools [60]. The in silico results supported previous findings: the binding of morphine (11) and naloxone (12) (Figure 3) into the hydrophobic pocket of MD-2 is TLR4-dependent. Binding of $\mathbf{1 1}$ induce the typical conformational change of MD-2 into its active form (agonist switch). In particular this is mediated by interaction of $\mathbf{1 1}$ with the Phe126 loop of MD-2 that confers stability to the subsequently formed TLR4/MD-2/11 complex. The interaction with TLR4 also stabilizes the MD-2/12 complex. However, 12 switches the Phe126 loop of MD-2 to its inactive (antagonist) conformation form. These data confirmed that subtle changes on the morphine structure can induce agonism to antagonism switch and that morphine scaffold could be used to generate potent and specific TLR4 modulators.

\section{Platycodin D}

Platycodin D (13; Figure 3), the major triterpene saponin in the root of Platycodon grandiflorum, exhibits a broad spectrum of anti-inflammatory effects by inhibiting LPS-induced TNF- $\alpha$ and IL-1 $\beta$ production [61] and NF- $\kappa \mathrm{B}$ activation [62]. The mechanism of action of $\mathbf{1 3}$ in protecting LPS-induced ALI has been recently studied and clarified [63]. In in vitro and in vivo models, $\mathbf{1 3}$ acts as a TLR4 antagonist by mediating the depletion of cholesterol from plasma membrane and therefore by reducing the translocation of TLR4 to lipid rafts. This mechanism was confirmed by the fact that cholesterol replenishment prevented 13-mediated TLR4 antagonism. Studies on raft-disrupting drugs supported these data, confirming that depletion of cholesterol can antagonize LPS-mediated TLR4 activation by the inhibition of TLR4 translocation into lipid rafts [64]. It has been reported that ethanol also acts by altering the LPS-induced redistribution of TLR 4 complex within the lipid raft, thus interfering with receptor clustering and subsequent signaling [65].

\section{P4-ATPases}

Phospholipids translocation from exoplasmic to cytoplasmic leaflet is mediated by integral membrane proteins, named P4-ATPases, which have a crucial function in the biogenesis of transport vesicles and in endocytic pathways $[66,67]$. The hypothesis that P4-ATPases (in particular CDC50A) can play a role in the TLR4 activation pathway has been recently investigated [68]. In this study, the LPS-mediated TLR4 activation has been analyzed by using CDC50A-depleted THP-1 and human monocyte-derived macrophages. LPS challenge of CDC50A-depleted THP-1 is responsible for a hyper-activation of the MyD88-dependent pathway, caused by the impaired endocytic TLR4 retrieval. There are two P4-ATPases (i.e., ATP8B1 and ATP11A) that are expressed in humans, and their deficiency is associated with severe chronic liver disease [69] and pulmonary disorders [70]. The exact contribution of P4-ATPases in TLR4-dependent inflammatory disease remains to be established. 


\section{Naringenin}

Naringenin (14; Figure 3) is a flavonoid naturally present in grapefruit and other citrus species that possess antiinflammatory and antioxidant activities, particularly important for inflammatory-associated atherosclerosis, arthritis and metabolic syndrome [71]. In a recent study, the underlying mechanism of the anti-inflammatory properties and life-protective efficacy of 14 in LPS-stimulated macrophages and in a murine endotoxaemia model was evaluated [72]. $\mathbf{1 4}$ was able to prevent TNF- $\alpha$ and IL- 6 upregulation, to inhibit NF- $\kappa$ B activation, and $\mathbf{1 4}$ was also responsible for the upregulated expression of ATF3 in LPS-stimulated murine macrophages. In lung tissues, the ATF3 expression is upregulated by the induction of AMPK that is provoked by 14. In murine models of endotoxaemia, 14 improved the proinflammatory reactions and the survival of mice. This AMPK-ATF3-dependent downregulation of the LPS/TLR 4 signaling pathway mediated by 14 represents a novel mechanism of TLR4 modulation.

\section{Nickel \& cobalt ions $\left(\mathrm{Ni}^{++} \& \mathrm{Co}^{++}\right)$}

Allergic contact dermatitis is a very common skin disease, and among over 3,000 allergens, nickel is one of the most common ones [73]. Recent findings revealed that hTLR4, but not TLR4 of other species including mouse, can be directly activated by nickel and cobalt ions [74]. This species-specific activation is due to the coordination of nickel or cobalt ions by a cluster of histidine residues on the ectodomain of hTLR 4 that is absent in the majority of other species TLR4. This study identified TLR 4 and MD-2 mutants not responsive to LPS that can be activated by nickel and cobalt ions. Starting from these observations, authors proposed a model for the activation of the TLR4/MD-2 complex mediated by transition metal bivalent cations. To enable TLR4 activation several interactions are required and interactions mediated by the histidine residues of the TLR4 ectodomains can be a driving force for TLR4 dimerization. This is confirmed by a study where a single TLR4 mutation (residue N433, important for the interactions of the two TLR 4 ectodomains), prevented the dimerization of TLR 4 in the presence of cobalt or nickel ions [75].

Interestingly, nickel and cobalt ions turned out to be able to induce the dimerization of TLR4 ectodomain in the absence of MD-2 in vitro in cell-free experiments. However, this effect was not observed in cells.

Lead $\left(\mathrm{Pb}^{++}\right)$

Lead $(\mathrm{Pb})$ exposure is a worldwide problem, and it has been shown that $\mathrm{Pb}$ exposure can impact the immune system integrity [76]. Pb neurotoxicity has been researched extensively, however, the proinflammatory role that this metal plays in the brain has not been fully understood. The inflammatory role of $\mathrm{Pb}$ has been recently presented [77], and experiments showed that exposure to $\mathrm{Pb}$ induce micro and astrogliosis by activating TLR4/MyD88/NF- $\mathrm{KB}$ signaling pathway. Increased levels of proinflammatory cytokines were also observed.

\section{Ferulic acid}

Ferulic acid (15; Figure 3) is a phenolic compound abundant in vegetables and fruits, and it presents several antioxidative and anti-inflammatory activities [78]. 15 pharmacological effects and the underlying mechanisms in mice with acetaminophen-induced hepatotoxicity have been investigated in a recent study [79]. 15 attenuated acetaminophen-induced serum TNF- $\alpha$ and IL- $1 \beta$ production, suppressed TLR 4 expression, and dampened MAPK and NF- $\mathrm{KB}$ activation. These data suggested that $\mathbf{1 5}$ is able to partially suppress TLR4-mediated inflammatory response. However, the molecular mechanism of action of $\mathbf{1 5}$ in relation with TLR4 still needs elucidation.

\section{Corilagin}

Corilagin (16; Figure 3) is a polyphenol isolated from the extract of Arctostaphylos uvaursi, it is identified in several plants and it presents anti-inflammatory and antibacterial activity [80]. A recent study found that $\mathbf{1 6}$ is able to inhibit both TLR4-dependent MyD88 and TRIF signaling pathways [81]. Cellular and animal models treated with LPS and 16 presented mRNA levels and expression of TLR4, MyD88, TRIF and TRAF6, as well as IL-5 and IL-1 $\beta$ cytokines levels significantly decreased compared with the group treated with LPS alone. The molecular mechanism of action of $\mathbf{1 6}$ still needs elucidation.

\section{Alpinetin}

Alpinetin (17; Figure 3) is a natural flavonoid with known antibacterial, antitumor and other therapeutic activities [82]. A recent study investigates the anti-inflammatory effect of $\mathbf{1 7}$ in dextran sulfate sodium-induced colitis in mice [83]. 17 pretreatment inhibited significantly the phosphorylation of IKKa/b, IkB $\alpha$ and p65 NF- $\mathrm{KB}$ activation, 
and TLR4 expression was downregulated in LPS-induced phorbol myristate acetate (PMA)-differentiated THP-1 cells. According to this study, $\mathbf{1 7}$ has a protective effect on dextran sulfate sodium-induced colitis and might be a promising therapeutic treatment. However, further research is required to understand the exact molecular mechanism of action of 17 .

\section{Epigallocatechin-3-gallate}

Green tea contains caffeine and polyphenolic compounds known as catechins. The most abundant catechol found in green tea is (-)-epigallocatechin-3-gallate (18; Figure 3) [84] which has been suggested to be responsible for many of the potential health effects of tea [82]. A recent study showed that $\mathbf{1 8}$ negatively regulates TLR4 pathway through the inhibition of downstream signaling [85]. 18 induced ubiquitination of TLR4, decreased TLR4 expression through E3 ubiquitin-protein ligase RNF216, and increased cGMP levels in macrophages. 18-induced TLR4 downregulation is completely canceled by the soluble guanylate cyclase (cGMP synthesis enzyme) inhibitor, and cGMP induction is sufficient to suppress TLR 4 expression. This is a novel mechanism for the downregulation of TLR 4 expression. To study the role of cGMP in downregulation of expression, TLR 4 could be particularly valuable.

\section{Celastrol}

Celastrol (19; Figure 3) is a pharmacologically active cyclic-penta-triterpene, extracted from the roots of the plant Tripterygium wilfordii. It has been reported that $\mathbf{1 9}$ is a potent immunosuppressive and anti-inflammatory agent [86]. A recent study investigated the protective effects of celastrol on TLR4-dependent liver injury in diabetic rats [87]. Diabetic rats presented steatohepatitis and proinflammatory cytokine significantly upregulated. 19-treated diabetic rats presented reduced hepatic inflammation and macrophages infiltration. TLR4, MyD88 and NF-KB expression, as well as downstream inflammatory factors IL-1 $\beta$ and TNF- $\alpha$ in the hepatic tissue of 19-treated rats were downregulated in a dose-dependent manner. Treatment with 19 delayed the progression of diabetic liver disease via the inhibition of TLR4/MyD88/NF- $\mathrm{kB}$ signaling pathways and its downstream inflammatory effectors. Further studies are required to clarify the molecular mechanisms of action of $\mathbf{1 9}$, and for the development of new treatments for diabetic liver injury.

\section{Conclusion}

After the failure of TLR4 antagonists Eritoran and TAK-242 to pass clinical trials as drugs against acute sepsis and septic shock, the most recent change of direction in TLR4-directed therapeutics is to use TLR 4 antagonist, included Eritoran, to block or reduce acute and chronic inflammatory diseases due to TLR4 activation by endogenous factors [88].

Another important paradigm change in the rational design of small-molecular TLR4 modulators is to move away from the structure of lipid A, the natural agonist, and explore new hit compounds derived from combinatorial libraries and natural sources (mainly plant secondary metabolites). Besides the necessity for companies and academic groups to have proprietary structures, the main reason to leave the ligand-based design so far adopted, is the low solubility in aqueous media of lipid A and lipid A-like molecules and their poor pharmacokinetic (adsorption and distribution) properties. Concerning synthetic molecules, new promising directions have been suggested in the use of nonlipid A-like synthetic glycolipids, as in the case of MGDG 1 and tri-MGCs 2 (Figure1) reported in this review [40,41]. This last type of antagonists show a new mechanism of action based on the targeting of membrane lipid rafts that would be essential to TLR4 organization in activated oligomeric species, and in the selective deprivation of CD14 co-receptor from plasma membrane [41]. Interestingly, a very similar mechanism of action based on membrane rafts targeting seems to be at the base of natural molecules such as the steroid 13 [63]. Membrane rafts disruption and impairment of CD14 function are new strategies for TLR 4 antagonism that could be used by other molecules and deserve to be investigated more in detail in the perspective to develop new generations of TLR 4 modulators. Phosphorylated lipo-monosaccharides as compound 3 [42,43], deriving from molecular simplification of lipid A, present several advantages in the perspective of industrial development, due to the much simpler synthesis compared with Eritoran, and retention of selectivity and antagonist potency. Innovative peptidomimetics (neoseptins) have been proposed in 2016 as interesting hit compounds to develop nonlipid A-like TLR4 agonists [46], while the low potency and the lack of activity on human TLR4 limit the applicability of these compounds. Other nonlipid A-like TLR4 modulators have been reviewed here among synthetic and natural molecules. In general, the main limitation of the studies on new TLR4 modulators reported in this review and 
Table 1. Selection of clinical trials testing drugs targeting TLR4.

\begin{tabular}{|c|c|c|c|c|}
\hline Compound & Action & Clinical phase & Indications & Ref./clinical trial identifier \\
\hline $\begin{array}{l}\text { MPL plus pollen (Pollinex } \\
\text { Quattro) }\end{array}$ & Agonist & Licensed & Allergy & [89] \\
\hline AS04 (Stimuvax) & Agonist & Licensed & Protection from HPV & [90] \\
\hline AS04 (Stimuvax) & Agonist & Phase II & $\begin{array}{l}\text { Rectal, prostate and colorectal } \\
\text { cancer }\end{array}$ & $\begin{array}{l}\text { NCT01507103, NCT01496131 and } \\
\text { NCT01462513 }\end{array}$ \\
\hline AV-411 (Ibudilast) & Antagonist & Phase II & $\begin{array}{l}\text { Asthma and poststroke } \\
\text { disorders }\end{array}$ & [91] \\
\hline $\mathrm{NI}-0101^{\dagger}$ & Antagonist & Phase II & Rheumatoid arthritis & [92] \\
\hline $\begin{array}{l}\text { GLA-SE (glucopyranosyl lipid } \\
\text { A-stable emulsion) }\end{array}$ & Agonist & Phase I & Metastatic sarcoma & NCT02180698 \\
\hline GSK1795091 & Agonist & Phase I & Cancer & NCT02798978 \\
\hline OM-174 (lipid A analog) & Agonist & Phase I & Solid tumor and melanoma & NCT01800812 and NCT01530698 \\
\hline E5564 (Eritoran) & Antagonist & Withdrawn & Severe sepsis & [13] \\
\hline TAK-242 (resatorvid) & Antagonist & Withdrawn & Severe sepsis & [30] \\
\hline
\end{tabular}

depicted in Figures 1-3 is the lack or very limited investigation of selectivity and toxicity. The TLR4 activity has been rarely compared with the activity on other TLRs or other biological targets, and toxicity studies are lacking as well.

\section{Future perspective}

Despite the majority of the natural and synthetic molecules reviewed here are in the early stage of preclinical development, ongoing clinical studies and a few licensed compounds demonstrate the potential of TLR4 modulators (Table 1). TLR4 agonists with a structure related to lipid A (MPL and AS04) have been licenced as vaccine adjuvants. Other lipid A mimetics (AS04, GLA-SE, GSK1795091 and OM-174) are in clinical Phase I or II as anticancer therapeutics. Small molecular TLR4 antagonist AV-411 (Ibudilast) is in Phase II clinical trials for the treatment of asthma and poststroke disorders. NI-0101 is the first anti-TLR4 monoclonal antibody to pass Phase I clinical trials for rheumatoid arthritis, showing safety and tolerability (Table 1).

Because of the very reduced number of chemicals in clinical phase of development as TLR4-based therapeutics, major efforts should be focused in the future to progress in the preclinical characterization of newly discovered hit compounds. To do this, complete data on toxicity and specificity should be collected for every new hit compound presented here. In parallel, it is still important to extend the chemical variety of TLR4 modulators by discovering new active molecules from natural sources (included fruits and vegetables) and from de novo designed synthetic molecules.

\section{Executive summary}

- TLR4 plays important roles in inflammation but also in repair processes after inflammation. TLR4 signal is activated by bacterial molecules and endogenous factors.

- TLR4 activators (agonists) and inhibitors (antagonists) are, respectively, potential vaccine adjuvants and antitumoral agents (agonists) and anti-inflammatory agents (antagonists).

- In the last 4 years several new synthetic TLR4 modulators have been discovered: some of them have a chemical structure related to lipid A, the natural TLR4 ligand produced by bacteria. Other synthetic molecules, such as neoseptins, Ugi compounds, chalcone and morphine derivatives, have a chemical structure not related to lipid A.

- Several natural compounds showed TLR4 activity: saturated fatty acids and oxidized phospholipids have agonist action.

- Other natural molecules recently discovered are active as TLR4 blockers (antagonists). These molecules are plant secondary metabolites: polyphenols, triterpenes and flavonoids.

- The TLR4-active compounds described in this review are in the early stage of preclinical development. Very few drug candidates targeting TLR4 are in clinical phase.

- It is necessary in the next future to increase the number of molecules that enter into clinical trials by characterizing the toxicity and specificity of action of the TLR4 modulators yet available. 


\section{Open access}

This work is licensed under the Attribution-NonCommercial-NoDerivatives 4.0 Unported License. To view a copy of this license, visit http://creativecommons.org/licenses/by-nc-nd/4.0/

\section{Acknowledgements}

The Italian Ministry for Foreign Affairs and International Cooperation (MAECI) is acknowledged.

\section{Financial \& competing interests disclosure}

This study was financially supported by the H2020-MSC-ETN-642157 project 'TOLLerant'. The authors have no other relevant affiliations or financial involvement with any organization or entity with a financial interest in or financial conflict with the subject matter or materials discussed in the manuscript apart from those disclosed.

No writing assistance was utilized in the production of this manuscript.

\section{References}

Papers of special note have been highlighted as: $\bullet$ of interest

1. Akira S, Takeda K. Toll-like receptor signalling. Nat. Rev. Immunol. 4(7), 499-511 (2004).

2. Knirel YA, Valvano MA. Bacterial lipopolysaccharides. Springer-Verlag Wien, Vienna, Austria (2011).

3. Molinaro A, Holst O, Di Lorenzo F et al. Chemistry of lipid A: at the heart of innate immunity. Chemistry 21(2), 500-519 (2015).

4. Yu L, Wang L, Chen S. Endogenous toll-like receptor ligands and their biological significance. J. Cell. Mol. Med. 14(11), 2592-2603 (2010).

5. Molteni M, Gemma S, Rossetti C. The role of toll-like receptor 4 in infectious and noninfectious inflammation. Mediators Inflamm. 2016, (2016).

6. Heneka MT, Carson MJ, El Khoury J et al. Neuroinflammation in Alzheimer's disease. Lancet Neurol. 14(4), 388-405 (2015).

7. Singer M, Deutschman CS, Seymour CW et al. The third international consensus definitions for sepsis and septic shock (Sepsis-3). JAMA 315(8), 801-810 (2016).

8. Fleischmann C, Scherag A, Adhikari NK et al. Assessment of global incidence and mortality of hospital-treated sepsis. Current estimates and limitations. Am. J. Respir. Crit. Care Med. 193(3), 259-272 (2016).

9. Keynan Y, Fowke KR, Ball TB, Meyers AFA. Toll-like receptors dysregulation after influenza virus infection: insights into pathogenesis of subsequent bacterial pneumonia. ISRN Pulmonology 2011, 6 (2011).

10. Shah NS, Greenberg JA, McNulty MC et al. Bacterial and viral co-infections complicating severe influenza: incidence and impact among 507 US patients, 2013-14. J. Clin. Virol. 80, 12-19 (2016).

11. Crum-Cianflone NF. Invasive aspergillosis associated with severe influenza infections. Open Forum Infect. Dis. 3(3), ofw171 (2016).

12. Shirey KA, Lai W, Scott AJ et al. The TLR4 antagonist, Eritoran, protects mice from lethal influenza infection. Nature 497(7450), 498-502 (2013).

13. Opal SM, Laterre PF, Francois B et al. Effect of Eritoran, an antagonist of MD2-TLR4, on mortality in patients with severe sepsis: the ACCESS randomized trial. JAMA 309(11), 1154-1162 (2013).

14. van Noort JM, Bsibsi M. Toll-like receptors in the CNS: implications for neurodegeneration and repair. Prog. Brain Res. 175, 139-148 (2009).

15. Hanamsagar R, Hanke ML, Kielian T. Toll-like receptor (TLR) and inflammasome actions in the central nervous system: new and emerging concepts. Trends Immunol. 33(7), 333-342 (2012).

16. Racila DM, Kline JN. Perspectives in asthma: molecular use of microbial products in asthma prevention and treatment. J. Allergy Clin. Immunol. 116(6), 1202-1205 (2005).

17. Herrick CA, Bottomly K. To respond or not to respond: T cells in allergic asthma. Nat. Rev. Immunol. 3(5), 405-412 (2003).

18. Peri F, Calabrese V. Toll-like receptor 4 (TLR4) modulation by synthetic and natural compounds: an update. J. Med. Chem. 57(9), 3612-3622 (2014).

19. Bachtell R, Hutchinson MR, Wang X, Rice KC, Maier SF, Watkins LR. Targeting the toll of drug abuse: the translational potential of toll-like receptor 4. CNS Neurol. Disord. Drug Targets 14(6), 692-699 (2015).

20. Wu MK, Huang TL, Huang KW, Huang YL, Hung YY. Association between toll-like receptor 4 expression and symptoms of major depressive disorder. Neuropsychiatr. Dis. Treat. 11, 1853-1857 (2015).

21. De Paola M, Sestito SE, Mariani A et al. Synthetic and natural small molecule TLR4 antagonists inhibit motoneuron death in cultures from ALS mouse model. Pharmacol. Res. 103, 180-187 (2016).

22. Thakur KK, Saini J, Mahajan K et al. Therapeutic implications of toll-like receptors in peripheral neuropathic pain. Pharmacol. Res. 115, 224-232 (2017). 
23. Bettoni I, Comelli F, Rossini C et al. Glial TLR4 receptor as new target to treat neuropathic pain: efficacy of a new receptor antagonist in a model of peripheral nerve injury in mice. Glia 56(12), 1312-1319 (2008).

24. Gioannini TL, Zhang D, Teghanemt A, Weiss JP. An essential role for albumin in the interaction of endotoxin with lipopolysaccharide-binding protein and sCD14 and resultant cell activation. J. Biol. Chem. 277(49), 47818-47825 (2002).

25. Wright SD, Ramos RA, Tobias PS, Ulevitch RJ, Mathison JC. CD14 a receptor for complexes of lipopolysaccharide (LPS) and LPS binding protein. Science 249(4975), 1431-1433 (1990).

26. Shimazu R, Akashi S, Ogata $\mathrm{H}$ et al. MD-2, a molecule that confers lipopolysaccharide responsiveness on toll-like receptor 4. J. Exp. Med. 189(11), 1777-1782 (1999).

27. Park BS, Song DH, Kim HM, Choi BS, Lee H, Lee JO. The structural basis of lipopolysaccharide recognition by the TLR4-MD-2 complex. Nature 458(7242), 1191-1195 (2009).

28. Gioannini TL, Teghanemt A, Zhang D, Levis EN, Weiss JP. Monomeric endotoxin:protein complexes are essential for TLR4-dependent cell activation. J. Endotoxin Res. 11(2), 117-123 (2005).

29. Huber M, Kalis C, Keck S et al. R-form LPS, the master key to the activation ofTLR4/MD-2-positive cells. Eur. J. Immunol. 36(3), 701-711 (2006).

30. Rice TW, Wheeler AP, Bernard GR et al. A randomized, double-blind, placebo-controlled trial of TAK-242 for the treatment of severe sepsis. Crit. Care Med. 38(8), 1685-1694 (2010).

31. Miyake K. Innate immune sensing of pathogens and danger signals by cell surface Toll-like receptors. Semin. Immunol. 19(1), 3-10 (2007).

32. Mancek-Keber M, Jerala R. Postulates for validating TLR4 agonists. Eur. J. Immunol. 45(2), 356-370 (2015).

- Critically discusses the conditions for the assessment of TLR4 activity of nonlipopolysaccharide agonists.

33. Li J, Csakai A, Jin J, Zhang F, Yin H. Therapeutic developments targeting toll-like receptor-4-mediated neuroinflammation. ChemMedChem. 11(2), 154-165 (2016).

34. Billod JM, Lacetera A, Guzman-Caldentey J, Martin-Santamaria S. Computational approaches to toll-like receptor 4 modulation. Molecules 21(8), (2016).

35. Rossignol DP, Lynn M. Antagonism of in vivo and ex vivo response to endotoxin by E5564, a synthetic lipid A analog. J. Endotoxin. Res. 8(6), 483-488 (2002).

36. Mata-Haro V, Cekic C, Martin M, Chilton PM, Casella CR, Mitchell TC. The vaccine adjuvant monophosphoryl lipid A as a TRIF-biased agonist of TLR4. Science 316(5831), 1628-1632 (2007).

37. Bowen WS, Minns LA, Johnson DA, Mitchell TC, Hutton MM, Evans JT. Selective TRIF-dependent signaling by a synthetic toll-like receptor 4 agonist. Sci. Signal. 5(211), ra13 (2012).

38. Peri F, Piazza M. Therapeutic targeting of innate immunity with toll-like receptor 4 (TLR4) antagonists. Biotechnol. Adv. 30(1), 251-260 (2012).

39. Liu X, Dong T, Zhou Y, Huang N, Lei X. Exploring the binding proteins of glycolipids with bifunctional chemical probes. Angew Chem. Int. Ed. Engl. 55(46), 14330-14334 (2016).

- An important chemical work on the synthesis and characterization of a glycolipid-based chemical probe.

40. Ulivi V, Lenti M, Gentili C, Marcolongo G, Cancedda R, Descalzi Cancedda F. Anti-inflammatory activity of monogalactosyldiacylglycerol in human articular cartilage in vitro: activation of an anti-inflammatory cyclooxygenase-2 (COX-2) pathway. Arthritis Res. Ther. 13(3), R92 (2011).

41. Flacher V, Neuberg P, Point $\mathrm{F}$ et al. Mannoside glycolipid conjugates display anti-inflammatory activity by inhibition of toll-like receptor-4 mediated cell activation. ACS Chem. Biol. 10(12), 2697-2705 (2015).

- This paper presents a new type of sugar-based TLR4 antagonists and investigates in detail their mechanism of action.

42. Cighetti R, Ciaramelli C, Sestito SE et al. Modulation of CD14 and TLR4.MD-2 activities by a synthetic lipid A mimetic. Chembiochem. 15(2), 250-258 (2014).

43. Perrin-Cocon L, Aublin-Gex A, Sestito SE et al. TLR4 antagonist FP7 inhibits LPS-induced cytokine production and glycolytic reprogramming in dendritic cells, and protects mice from lethal influenza infection. Sci. Rep. 7, 40791 (2017).

44. Shirey KA, Lai W, Scott AJ et al. The TLR4 antagonist Eritoran protects mice from lethal influenza infection. Nature 497(7450), 498-502 (2013).

- First showed that synthetic TLR4 antagonist can be used to block TLR4-dependent influenza lethality.

45. Yamamoto H, Oda M, Kanno M et al. Chemical hybridization of vizantin and lipid A to generate a novel LPS antagonist. Chem. Pharm. Bull. (Tokyo) 64(3), 246-257 (2016).

46. Morin MD, Wang Y, Jones BT et al. Discovery and structure-activity relationships of the neoseptins: a new class of toll-like receptor-4 (TLR4) agonists. J. Med. Chem. 59(10), 4812-4830 (2016).

47. Marshall JD, Heeke DS, Rao E et al. A novel class of small molecule agonists with preference for human over mouse TLR4 activation. PLoS ONE 11(10), (2016). 
48. Kuronuma K, Mitsuzawa $\mathrm{H}$, Takeda $\mathrm{K}$ et al. Anionic pulmonary surfactant phospholipids inhibit inflammatory responses from alveolar macrophages and U937 cells by binding the lipopolysaccharide-interacting proteins CD14 and MD-2. J. Biol. Chem. 284(38), 25488-25500 (2009).

49. Kandasamy P, Numata M, Berry KZ et al. Structural analogs of pulmonary surfactant phosphatidylglycerol inhibit toll-like receptor 2 and 4 signaling. J. Lipid Res. 57(6), 993-1005 (2016).

50. Zhang Y, Wu J, Ying S et al. Discovery of new MD2 inhibitor from chalcone derivatives with anti-inflammatory effects in LPS-induced acute lung injury. Sci. Rep. 6, 25130 (2016).

51. Peluso MR, Miranda CL, Hobbs DJ, Proteau RR, Stevens JF. Xanthohumol and related prenylated flavonoids inhibit inflammatory cytokine production in LPS-activated THP-1 monocytes: structure-activity relationships and in silico binding to myeloid differentiation protein-2 (MD-2). Planta Med. 76(14), 1536-1543 (2010).

52. Rodrigues-Diez R, González-Guerrero C, Ocaña-Salceda C et al. Calcineurin inhibitors cyclosporine A and tacrolimus induce vascular inflammation and endothelial activation through TLR4 signaling. Sci. Rep. doi: 10.1038/srep27915. (2016).

53. Xiang N, Liu J, Liao Y et al. Abrogating ClC-3 inhibits LPS-induced inflammation via blocking the TLR4/NF-kB pathway. Sci. Rep. doi:10.1038/srep27583 (2016) (Epub ahead of print).

54. Selfridge BR, Wang X, Zhang Y et al. Structure-activity relationships of (+)-naltrexone-inspired toll-like receptor 4 (TLR4) antagonists. J. Med. Chem. 58(12), 5038-5052 (2015).

55. Chen CY. TCM Database@Taiwan: the world's largest traditional Chinese medicine database for drug screening in silico. PLoS ONE 6(1), e15939 (2011).

56. Jeong E, Lee JY. Intrinsic and extrinsic regulation of innate immune receptors. Yonsei Med. J. 52(3), 379-392 (2011).

57. Wang Y, Qian Y, Fang Q et al. Saturated palmitic acid induces myocardial inflammatory injuries through direct binding to TLR4 accessory protein MD2. Nat. Commun. doi:10.1038/ncomms13997 (2017).

58. Huang S, Rutkowsky JM, Snodgrass RG et al. Saturated fatty acids activate TLR-mediated proinflammatory signaling pathways. J. Lipid Res. 53(9), 2002-2013 (2012).

59. Wang X, Loram LC, Ramos K et al. Morphine activates neuroinflammation in a manner parallel to endotoxin. Proc. Natl Acad. Sci. USA 109(16), 6325-6330 (2012).

60. Shah M, Anwar MA, Yesudhas D, Krishnan J, Choi S. A structural insight into the negative effects of opioids in analgesia by modulating the TLR4 signaling: an in silico approach. Sci. Rep. doi:10.1038/srep39271 (2016).

61. Wang C, Schuller Levis GB, Lee EB et al. Platycodin D and D3 isolated from the root of Platycodon grandiflorum modulate the production of nitric oxide and secretion of TNF-alpha in activated RAW 264.7 cells. Int. Immunopharmacol. 4(8), 1039-1049 (2004).

62. Chung JW, Noh EJ, Zhao HL et al. Anti-inflammatory activity of prosapogenin methyl ester of platycodin D via nuclear factor-kappaB pathway inhibition. Biol. Pharm. Bull. 31(11), 2114-2120 (2008).

63. Hu X, Fu Y, Lu X et al. Protective effects of platycodin D on lipopolysaccharide-induced acute lung injury by activating LXR $\alpha-\mathrm{ABCA1}$ signaling pathway. Front. Immunol. 7, 644 (2016).

64. Olsson S, Sundler R. The role of lipid rafts in LPS-induced signaling in a macrophage cell line. Mol. Immunol. 43(6), 607-612 (2006).

65. Szabo G, Dolganiuc A, Dai Q, Pruett SB. TLR4, ethanol, and lipid rafts: a new mechanism of ethanol action with implications for other receptor-mediated effects. J. Immunol. 178(3), 1243-1249 (2007).

66. Lopez-Marques RL, Theorin L, Palmgren MG, Pomorski TG. P4-ATPases: lipid flippases in cell membranes. Pflugers Arch. 466(7), 1227-1240 (2014).

67. van der Mark VA, Elferink RP, Paulusma CC. P4 ATPases: flippases in health and disease. Int. J. Mol. Sci. 14(4), 7897-7922 (2013).

68. van der Mark VA, Ghiboub M, Marsman C et al. Phospholipid flippases attenuate LPS-induced TLR4 signaling by mediating endocytic retrieval of Toll-like receptor 4. Cell Mol. Life Sci. 74(4), 715-730 (2017).

69. Pawlikowska L, Strautnieks S, Jankowska I et al. Differences in presentation and progression between severe FIC1 and BSEP deficiencies. J. Hepatol. 53(1), 170-178 (2010).

70. Fingerlin TE, Murphy E, Zhang W et al. Genome-wide association study identifies multiple susceptibility loci for pulmonary fibrosis. Nat. Genet. 45(6), 613-620 (2013).

71. Mulvihill EE, Burke AC, Huff MW. Citrus flavonoids as regulators of lipoprotein metabolism and atherosclerosis. Annu. Rev. Nutr. 36, 275-299 (2016).

72. Liu X, Wang N, Fan $\mathrm{S}$ et al. The citrus flavonoid naringenin confers protection in a murine endotoxaemia model through AMPK-ATF3-dependent negative regulation of the TLR4 signalling pathway. Sci. Rep. 6, 39735 (2016).

73. Vocanson M, Hennino A, Rozieres A, Poyet G, Nicolas JF. Effector and regulatory mechanisms in allergic contact dermatitis. Allergy 64(12), 1699-1714 (2009).

74. Oblak A, Pohar J, Jerala R. MD-2 determinants of nickel and cobalt-mediated activation of human TLR4. PLoS ONE 10(3), e0120583 (2015). 
75. Raghavan B, Martin SF, Esser PR, Goebeler M, Schmidt M. Metal allergens nickel and cobalt facilitate TLR4 homodimerization independently of MD2. EMBO Rep. 13(12), 1109-1115 (2012).

76. Bunn TL, Parsons PJ, Kao E, Dietert RR. Exposure to lead during critical windows of embryonic development: differential immunotoxic outcome based on stage of exposure and gender. Toxicol. Sci. 64(1), 57-66 (2001).

77. Chibowska K, Baranowska-Bosiacka I, Falkowska A, Gutowska I, Goschorska M, Chlubek D. Effect of lead (Pb) on inflammatory processes in the brain Int. J. Mol. Sci. 17(12), 2140 (2016).

78. Das $\mathrm{U}$, Manna $\mathrm{K}$, Sinha $\mathrm{M}$ et al. Role of ferulic acid in the amelioration of ionizing radiation induced inflammation: a murine model. PLoS ONE 9(5), e97599 (2014).

79. Yuan J, Ge K, Mu J et al. Ferulic acid attenuated acetaminophen-induced hepatotoxicity though down-regulating the cytochrome P 2E1 and inhibiting toll-like receptor 4 signaling-mediated inflammation in mice. Am. J. Transl. Res. 8(10), 4205-4214 (2016).

80. Dang YP, Chen YF, Li YQ, Zhao L. Developments of anticoagulants and new agents with anti-coagulant effects in deep vein thrombosis. Mini Rev. Med. Chem. 17(4), 338-350 (2017).

81. Li HR, Liu J, Zhang SL et al. Corilagin ameliorates the extreme inflammatory status in sepsis through TLR4 signaling pathways. BMC Complement Altern. Med. 17, 18 (2017).

82. Wolfram S, Wang Y, Thielecke F. Anti-obesity effects of green tea: from bedside to bench. Mol. Nutr. Food Res. 50(2), 176-187 (2006).

83. He X, Wei Z, Wang J et al. Alpinetin attenuates inflammatory responses by suppressing TLR4 and NLRP3 signaling pathways in DSS-induced acute colitis. Sci. Rep. 6, 28370 (2016).

84. Kovacs EM, Lejeune MP, Nijs I, Westerterp-Plantenga MS. Effects of green tea on weight maintenance after body-weight loss. Br. J. Nutr. 91(3), 431-437 (2004).

85. Kumazoe M, Nakamura Y, Yamashita M et al. Green tea polyphenol epigallocatechin-3-gallate suppresses toll-like receptor 4 expression via upregulation of E3 ubiquitin-protein ligase RNF216. J. Biol. Chem. 292(10), 4077-4088 (2017).

86. Shrivastava S, Jeengar MK, Reddy VS, Reddy GB, Naidu VG. Anticancer effect of celastrol on human triple negative breast cancer: possible involvement of oxidative stress, mitochondrial dysfunction, apoptosis and PI3K/Akt pathways. Exp. Mol. Pathol. 98(3), 313-327 (2015).

87. Han L, Li C, Sun B et al. Protective effects of celastrol on diabetic liver injury via TLR4/MyD88/NF- $\mathrm{B}$ signaling pathway in type 2 diabetic rats. J. Diabetes Res. 2016, (2016).

88. Dickinson SE, Wondrak GT. TLR4-directed molecular strategies targeting skin photodamage and carcinogenesis. Curr. Med. Chem. doi:10.2174/0929867324666170828125328 (2017) (Epub ahead of print).

89. DuBuske LM, Castells M, Holdich T. Significant reduction in combined symptom and medication score compared with placebo following MPL-adjuvanted uSCIT in patients with seasonal grass pollen allergy. J. Allergy Clin. Immunol. 123(Suppl. 2), S216 (2009).

90. Romanowski B, Schwarz TF, Ferguson L et al. Sustained immunogenicity of the HPV-16/18 AS04-adjuvanted vaccine administered as a two-dose schedule in adolescent girls: five-year clinical data and modeling predictions from a randomized study. Hum. Vaccin. Immunother. 12(1), 20-29 (2016).

91. Jia ZJ, Wu FX, Huang QH, Liu JM. [Toll-like receptor 4: the potential therapeutic target for neuropathic pain]. Zhongguo Yi Xue Ke Xue Yuan Xue Bao 34(2), 168-173 (2012).

92. Monnet E, Lapeyre G, Poelgeest EV et al. Evidence of NI-0101 pharmacological activity, an anti-TLR4 antibody, in a randomized Phase I dose escalation study in healthy volunteers receiving LPS. Clin. Pharmacol. Ther. 101(2), 200-208 (2017). 